\title{
WAVELET FOLDING AND DECORRELATION ACROSS THE SCALE
}

\author{
J. Tian, R. G. Baraniuk, R. O. Wells, Jr., \\ Computational Mathematics Laboratory \\ Rice University \\ Houston, TX 77005, USA \\ juntian,richb,wells@rice.edu
}

\author{
D. M. Tan and H. R. Wu
School of Computer Science and Software Engineering Monash University, Australia dmt,hrw@csse.monash.edu.au

\begin{abstract}
The discrete wavelet transform (DWT) gives a compact multiscale representation of signals and provides a hierarchical structure for signal processing. It has been assumed the DWT can fairly well decorrelate real-world signals. However a residual dependency structure still remains between wavelet coefficients. It has been observed magnitudes of wavelet coefficients are highly correlated, both across the scale and at neighboring spatial locations. In this paper we present a wavelet folding technique, which folds wavelet coefficients across the scale and removes the across-the-scale dependence to a larger extent. It produces an even more compact signal representation and the energy is more concentrated in a few large coefficients. It has a great potential in applications such as image compression.
\end{abstract}

\section{INTRODUCTION}

Ever since the terminology "wavelet" was first introduced, in the context of a mathematical transform, by A. Grossmann and J. Morlet [1] in 1984, wavelet theory and its applications have grown tremendously. The discrete wavelet transform (DWT) and its fast implementation was introduced by S. G. Mallat [2] in 1989. For a discrete signal $x=\left\{x_{n}\right\}$, the DWT of $x$ consists of two parts, the low frequency part (scaling coefficients) $L=\left\{L_{k}\right\}$ and the high frequency (wavelet coefficients) part $H=\left\{H_{k}\right\}$,

$$
\begin{aligned}
L_{k} & =\sum_{n \in \mathbf{Z}} h_{n-2 k} x_{n}, \\
H_{k} & =\sum_{n \in \mathbf{Z}} g_{n-2 k} x_{n},
\end{aligned}
$$

where $h$ and $g$ are the (analysis) scaling filter and wavelet filter, respectively. The $L$ component can be further

J. Tian, R. G. Baraniuk, and R. O. Wells, Jr. were supported in part by AFOSR/DARPA, Exxon Production Research, NSF, and Northrop Grumman Corporation. Web sites: www.cml.rice.edu, www.csse.monash.edu.au decomposed into its low and high frequency parts, and so on, to produce a multiscale representation of the original signal $x$. From $L$ and $H$, the inverse discrete wavelet transform (IDWT) will reconstruct $x$ as

$$
x_{n}=\sum_{k \in \mathbf{Z}}\left(\widetilde{h}_{n-2 k} L_{k}+\widetilde{g}_{n-2 k} H_{k}\right),
$$

where $\widetilde{h}$ and $\widetilde{g}$ are the synthesis scaling filter and wavelet filter, respectively. In an orthogonal case, $h=\widetilde{h}, g=\widetilde{g}$.

The success of the DWT can be attributed to its three primary properties [3]:

Locality: Each wavelet coefficient is localized simultaneously in time and frequency.

Multiresolution: Wavelet coefficients are compressed and dilated to analyze at a nested set of scales.

Compression: The wavelet transforms of real-world signals tend to be sparse.

Based on the above three properties, it has been assumed that the DWT can decorrelate real-world signals fairly well. However a residual dependency structure still remains between wavelet coefficients. It has been observed $[3,4,5,6,7,8,9]$ that magnitudes of wavelet coefficients are actually highly correlated, i.e., large values of wavelet coefficients tend to propagate at the same relative spatial locations across the scale, and at neighboring spatial locations at the same scale. In this paper we present a wavelet folding technique, which folds wavelet coefficients across the scale and removes the across-the-scale dependence to a larger extent.

This paper is organized as follows. In Section 2 we give a brief overview of a data folding method for the discrete cosine transform (DCT). We introduce the wavelet folding technique in Section 3. Beginning with a simple example, we compute the wavelet packet of the data and construct a wavelet folding matrix. Then we apply the DWT on the scale direction of this matrix 
to obtain a more compact representation. We conclude the paper in Section 4.

\section{DATA FOLDING FOR DCT}

A data folding method for the DCT was presented in [10]. It rearranges the data to allow the practical application of the DCT to expand into higher dimensions. Let us explain the data folding method with an example.

Assume we have a $128 \times 128$ image with pixel values $a_{i, j}, 0 \leq i, j \leq 127$. The standard two-dimensional (2D) DCT is applied to blocks of the image with block size $8 \times 8$, i.e., for each pair $(k, l), 0 \leq k, l \leq 15$, we apply DCT on the $8 \times 8$ matrix $A_{k, l}=\left(a_{8 k+i, 8 l+j}\right), 0 \leq$ $i, j \leq 7$. The DCT coefficients of $A_{k, l}$ is still an $8 \times 8$ matrix, which is denoted as $D_{k, l}=\left(d_{8 k+i, 8 l+j}\right), 0 \leq$ $i, j \leq 7$. The data folding method rearranges the $2-\bar{D}$ DCT coefficients $D=\left(d_{8 k+i, 8 l+j}\right), 0 \leq k, l \leq 15,0 \leq$ $i, j \leq 7$, to a new four-dimensional (4-D) matrix

$$
\mathcal{D}:=\left(d_{k, l, i, j}\right), \quad 0 \leq k, l \leq 15, \quad 0 \leq i, j \leq 7,
$$

and take another 2-D DCT of this new matrix $\mathcal{D}$ along the $k$ and $l$ directions. Figure 1 illustrates the folding of a 2-D $128 \times 128$ image into a 4-D $16 \times 16 \times 8 \times 8$ data array for a 4-D DCT. The statistical assumption behind the data folding method is that it is very likely that high correlation exists between the post DCT blocks, that is, DCT coefficients at the same location (the $i, j$ coordinates for $0 \leq i, j \leq 7$ ) between different blocks (the $k, l$ coordinates). Therefore, it may be desirable to fold the DC'T coefficients from different blocks and apply another 2-D DCT along the $k$ and $l$ directions to remove further spatial redundancies. The data folding method has been applied to image coding and video coding. Experimental results show that a hybrid coding algorithm using the data folding method outperforms the standard 2-D block DCT method for most of the test images. For more details of the data folding method, we refer to [10].

\section{WAVELET FOLDING}

Similar to the DCT, wavelet coefficients are not statistically independent. Figure 2 is a seven-scale wavelet transform of a bump signal in [11]. The bump signal lies atop the time-frequency tiling provided by the DWT. Each tile is colored as a monotonic function of the wavelet coefficient magnitude, with darker tiles indicating larger magnitude. As we can see, large magnitude coefficients (dark colored) tend to occur at the same relative spatial locations across the scale at different subbands, which we call the "persistence across
$8 \times 8$ pixel sub-blocks

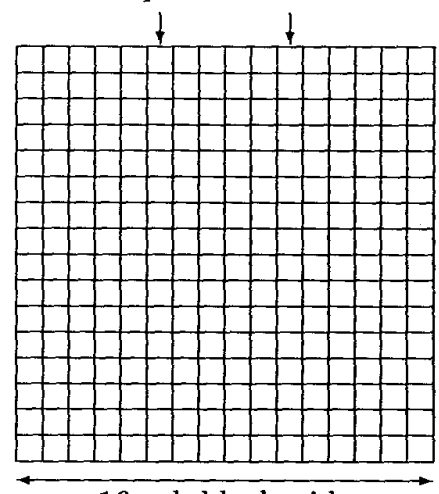

16 sub-block wide

Figure 1: A 2-D Data Folding for 4-D DCT

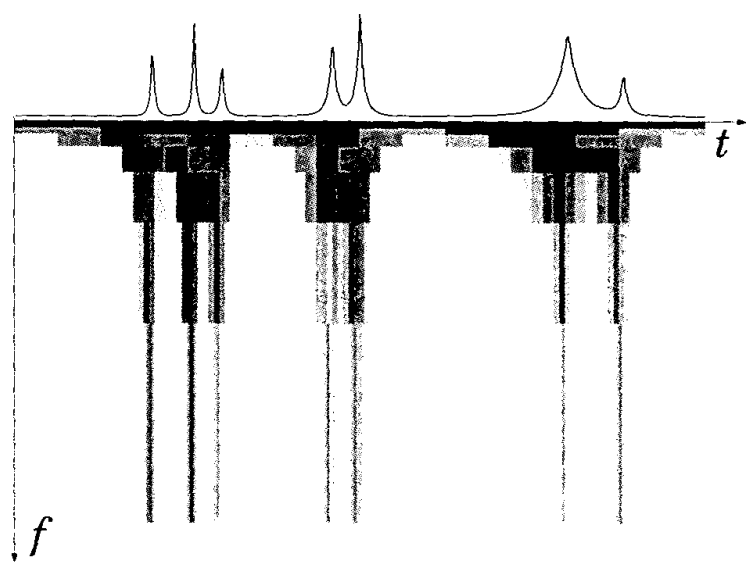

Figure 2: Persistence Across Scale

scale" property. There is also strong evidence of clustering (dependence at neighboring spatial locations at the same scale), but we will not address the clustering problem in this paper.

Coming back to the "persistence across scale" property of wavelet coefficients, let us denote by $L^{j}=\left\{L_{k}^{j}\right\}$ and $H^{j}=\left\{H_{k}^{j}\right\}$ as the scaling coefficients and the wavelet coefficients, at $j$-th scale, of a discrete signal $x=\left\{x_{n}\right\}$, respectively,

$$
\begin{aligned}
L_{k}^{j} & =\sum_{n \in \mathbf{Z}} h_{n-2 k} L_{n}^{j-1}, \\
H_{k}^{j} & =\sum_{n \in \mathbf{Z}} g_{n-2 k} L_{n}^{j-1}
\end{aligned}
$$

with $L^{0}=x$. We are interested in exploring the dependence among $H^{1}, H^{2}, H^{3}, \cdots$. 
Let us assume for now that $h$ and $g$ are the Haar scaling filter and wavelet filter,

$$
h=\{1 / \sqrt{2}, 1 / \sqrt{2}\}, \quad g=\{-1 / \sqrt{2}, 1 / \sqrt{2}\},
$$

and $x$ is the square sequence $\{0,1,4,9,16,25,36, \cdots\}$. Thus

$$
\begin{aligned}
H^{1} & =\{1 / \sqrt{2}, 5 / \sqrt{2}, 9 / \sqrt{2}, 13 / \sqrt{2}, 17 / \sqrt{2}, \cdots\}, \\
L^{1} & =\{1 / \sqrt{2}, 13 / \sqrt{2}, 41 / \sqrt{2}, 85 / \sqrt{2}, 145 / \sqrt{2}, \cdots\}, \\
H^{2} & =\{6,22,38,54,70,86,102, \cdots\} .
\end{aligned}
$$

Comparing $H^{1}$ and $H^{2}$, they are both arithmetic series. What is striking behind it is that if we take another DWT on $H^{1}$ (which is the wavelet packet [12] formulated by R. R. Coifman, Y. Meyer, and M. V. Wickerhauser), the scaling coefficients $L$ of $H^{1}$ is given by

$$
L\left(H^{1}\right)=\{3,11,19,27,35,43,51, \cdots\},
$$

which is exactly $H^{2}$ divided by 2 !

The DWT of Haar scaling filter and wavelet filter could only remove the constant (which is equivalent to the zeroth vanishing moment) in the data. However by combining the wavelet coefficients at two adjacent scales, one can remove the quadratic relation (which is the second vanishing moment) in the data, as illustrated above.

The above simple example leads to the introduction of wavelet folding. Let us begin with the standard DWT. Because of the downsampling procedure in the filtering by $h$ and $g$, the sizes of wavelet coefficients at different scales $H^{1}, H^{2}, H^{3}, \cdots, H^{j}, \cdots$, are different by a factor of $2,\left|H^{1}\right|=2\left|H^{2}\right|=4\left|H^{3}\right|=\cdots=$ $2^{j-1}\left|H^{j}\right|=\cdots$. The wavelet packet comes naturally to reduce the sizes of $H \mathrm{~s}$ at finer scales (the $H^{j}$ with small $j$ ) so that they will have the same size for the folding processing. Unlike the DWT which only decomposes the scaling coefficients $L$, the wavelet packet decomposes the wavelet coefficients $H$ as well. So for the wavelet coefficients $H^{j}$ at the $j$-th scale, one can have

$$
\begin{aligned}
& L_{k}\left(H^{j}\right)=\sum_{n \in \mathbf{Z}} h_{n-2 k} H_{n}^{j}, \\
& H_{k}\left(H^{j}\right)=\sum_{n \in \mathbf{Z}} g_{n-2 k} H_{n}^{j} .
\end{aligned}
$$

One could further decompose the $L\left(H^{j}\right)$ and $H\left(H^{j}\right)$ by filtering with $h$ and $g$, and so on, until some desired decomposition structure is reached. For our wavelet folding purpose, we will only further decompose $L\left(H^{j}\right)$ until $L\left(L \cdots L\left(H^{j}\right)\right)$ having the same size as $H^{j+m}$, the wavelet coefficients at some scale $j+m$. Figure 3 illustrate a three scale wavelet folding decomposition,

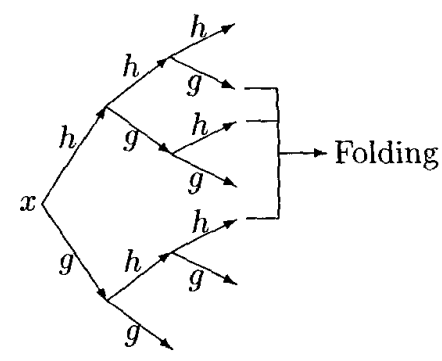

Figure 3: Three Scale Wavelet Folding

with $m=2$, and $L\left(L\left(H^{1}\right)\right), L\left(H^{2}\right), H^{3}$ have the same size for the folding processing. For convenience, denote

$$
L^{(n)}\left(H^{j}\right)=: \underbrace{L L \cdots L}_{n}\left(H^{j}\right) .
$$

Our goal is to further reduce the dependence among $H^{j}, H^{j+1}, \cdots, H^{j+m}$. Now $L^{(m)}\left(H^{j}\right), L^{(m-1)}\left(H^{j+1}\right)$, $\cdots, L^{(1)}\left(H^{j+m-1}\right), H^{j+m}$ all have the same size as $\left|H^{j+m}\right|$. Construct a new wavelet folding matrix with $L^{(m)}\left(H^{j}\right), L^{(m-1)}\left(H^{j+1}\right), \cdots, L^{(1)}\left(H^{j+m-1}\right), H^{j+m}$ as its $(m+1)$ rows. Along each column of the wavelet folding matrix, compute a wavelet coefficient $w_{k}$ by filtering with some wavelet filter $g^{\prime}$, where $k$ is the column index. Note that we do not compute the scaling coefficients, since we are trying to remove the across-thescale dependence by taking the difference (the wavelet coefficient). Also note we compute only one wavelet coefficient for each column. With this wavelet coefficient $w_{k}$, the coefficient of $H^{j+m}$ at the $k$-th column in the wavelet folding matrix can be retrieved from other $m$ rows $L^{(m)}\left(H^{j}\right), L^{(m-1)}\left(H^{j+1}\right), \cdots, L^{(1)}\left(H^{j+m-1}\right)$, since the filtering is a linear transform. Thus the wavelet coefficients $H^{j+m}$ can be fully retrieved from the sequence $w=\left\{w_{k}\right\}$ and $L^{(m)}\left(H^{j}\right), L^{(m-1)}\left(H^{j+1}\right), \cdots$, $L^{(1)}\left(H^{j+m-1}\right)$. So given $H^{j}, H^{j+1}, \cdots, H^{j+m-1}$, the wavelet coefficients at $(j+m)$-th scale, $H^{j+m}$, can be retrieved from the new sequence $w$. It is also clear now that the value of $m$ should be equal to the filter length $\left|g^{\prime}\right|$. We claim that the sequence $w$ is a more compact representation than the wavelet coefficients $H^{j+m}$, because of the following.

Theorem 1 For a sequence $x=\left\{x_{n} \mid x_{n}=a n^{2}+b n+\right.$ $c\}$, where $a, b, c$ are some constants independent of $n$, assume $h$ and $g$ are the Haar scaling filter and wavelet filter, then

$$
H^{2}(x)=2 L\left(H^{1}(x)\right) .
$$

Therefore, by taking another DWT along the column on the wavelet folding matrix consisting of $H^{2}$ 
and $L\left(H^{1}\right)$, the new sequence $w$ will be a zero sequence (the factor 2 comes from the normalization factor $\sqrt{2}$ of the scaling filter and wavelet filter). Thus using the wavelet folding, one can remove the quadratic relation in the data by the Haar scaling filter and wavelet filter, which usually requires longer filters (for example, the D6 filter (13] with length 6) in a standard DWT.

Moving beyond the Haar, we have

Theorem 2 Assume the wavelet filters $g$ and $g^{\prime}$ have vanishing moments up to order $N$ and $N^{\prime}$, respectively, then the wavelet folding technique can remove the polynomial relation in the data up to order $N+N^{\prime}+2$.

Due to the page limit, we have omitted the proofs of two theorems presented in this paper. The proofs can be found in [14]. Again because of the normalization factor $\sqrt{2}$, one needs to enlarge the magnitudes of $L^{(n)}\left(H^{j}\right)$ before the DWT across the scale.

\section{CONCLUSIONS}

In this paper, we introduce a new wavelet folding technique which reduces the across-the-scale dependence to a larger extent. Mathematically, we are looking at the dependence among the operators

$$
\begin{gathered}
(D \circ g) \circ(D \circ h) \circ(D \circ h) \cdots(D \circ h) \\
(D \circ h) \circ(D \circ g) \circ(D \circ h) \cdots(D \circ h) \\
\vdots \\
(D \circ h) \circ(D \circ h) \circ(D \circ h) \cdots(D \circ g)
\end{gathered}
$$

where each operator has exactly $(n-1) h$ operations, one $g$ operation, and $n D$ operations. Here $D$ represents the downsampling, $h$ and $g$ the convolutions with the reversed filters $h$ and $g$ (i.e., $h_{-n}, g_{-n}$ ), respectively. Since $h, g, D$ do not commute, these operators are all different. The wavelet folding technique gives a linear approximation to reduce the dependence. In practice, one may choose a smaller $m$ for consideration of execution time and memory overhead, instead of setting $m$ to be the full tree depth.

\section{REFERENCES}

[1] A. Grossmann and J. Morlet. Decomposition of Hardy functions into square integrable wavelets of constant shape. SIAM J. Math. Anal., 15(4):723736, July 1984.

[2] S. G. Mallat. Multiresolution approximation and wavelet orthonormal bases of $L^{2}(\mathbf{R})$. Trans. $A M S$, 315(1):69-87, September 1989.
[3] M. S. Crouse, R. D. Nowak, and R. G. Baraniuk. Wavelet-based statistical signal processing using hidden Markov models. IEEE Trans. Signal Processing, 46:886-902, 1998.

[4] R. W. Buccigrossi and E. P. Simoncelli. Image compression via joint statistical characterization in the wavelet domain. IEEE Trans. Image Processing, 1999. To appear.

[5] S. G. Mallat and W. Hwang. Singularity detection and processing with wavelets. IEEE Trans. Inform. Theory, 38(2):617-643, 1992.

[6] P. Müller and B. Vidakovic, editors. Bayesian Inference in Wavelet Based Models. Springer-Verlag, 1999.

[7] M. T. Orchard and K. Ramchandran. An investigation of wavelet-based image coding using an entropy-constrained quantization framework. In Proc. Data Compression Conference, pages 341$350,1994$.

[8] E. Ordentlich, M. Weinberger, and G. Seroussi. A low-complexity modeling approach for embedded coding of wavelet coefficients. In Proc. Data Compression Conference, pages 408-417, 1998.

[9] J. M. Shapiro. Embedded image coding using zerotrees of wavelet coefficients. IEEE Trans. Signal Processing, 41:3445-3462, December 1993.

[10] D. M. Tan and H. R. Wu. Multi-dimensional discrete cosine transform for image compression. In Proc. 6th IEEE International Conference on Electronics, Circuits and Systems, pages T1E2.1T1E2.5, Cyprus, September 1999.

[11] D. Donoho and I. Johnstone. Adapting to unknown smoothness via wavelet shrinkage. J. Amer. Stat. Assoc., 90:1200-1224, December 1995.

[12] R. R. Coifman, Y. Meyer, and M. V. Wickerhauser. Size properties of wavelet packets. In Ruskai et al., editor, Wavelets and their Applications, pages 453-470. Jones and Bartlett, 1992.

[13] I. Daubechies. Orthonormal bases of compactly supported wavelets. Comm. Pure Appl. Math., XLI:906-966, 1988.

[14] J. Tian, R. G. Baraniuk, and R. O. Wells, Jr. Wavelet folding, vanishing moments, and polynomial representation. Technical Report CML TR-9910, Computational Mathematics Laboratory, Rice University, October 1999. 